\title{
Noise spectroscopy and interlayer phase-coherence in bilayer quantum Hall systems
}

\author{
Yogesh N. Joglekar ${ }^{1}$, Alexander V. Balatsky ${ }^{1}$ and Allan H. MacDonald ${ }^{2}$ \\ ${ }^{1}$ Theoretical Division, Los Alamos National Laboratory, Los Alamos, New Mexico 87544. \\ ${ }^{2}$ Department of Physics, University of Texas at Austin, Austin, Texas 78712.
}

(Dated: April 7, 2018)

\begin{abstract}
Bilayer quantum Hall systems develop strong interlayer phase-coherence when the distance between layers is comparable to the typical distance between electrons within a layer. The phasecoherent state has until now been investigated primarily via transport measurements. We argue here that interlayer current and charge-imbalance noise studies in these systems will be able to address some of the key experimental questions. We show that the characteristic frequency of current-noise is that of the zero wavevector collective mode, which is sensitive to the degree of order in the system. Local electric potential noise measured in a plane above the bilayer system on the other hand is sensitive to finite-wavevector collective modes and hence to the soft-magnetoroton picture of the order-disorder phase transition.
\end{abstract}

PACS numbers: 73.21.-b

Introduction: Bilayer quantum Hall systems near total filling factor $\nu=1$ undergo a quantum phase transition from a compressible state to an incompressible state with interlayer phase-coherence as the ratio of layer separation to magnetic length $d / l$ is reduced [1]. This phasecoherent state survives in the limit of vanishing interlayer tunneling $\Delta_{t}$, in which case the charge-gap in the phase-coherent state arises purely because of electronelectron interactions between the two layers. The incompressible state can be regarded either as an easy-plane ferromagnet [2, 3, 4, 5] or as excitonic superfluid [6]. The estimated tunneling amplitude in the experimental samples, $\Delta_{t} \sim 50 \mu \mathrm{K}$, is much smaller than any other energy-scale [], suggesting that interlayer phasecoherence is established spontaneously when the ratio $d / l$ is smaller than some critical value $d_{c r} / l$. Transport measurements in these systems change spectacularly when the compressible-to-incompressible phase boundary is crossed [7, 8] and can largely be explained in terms of excitonic condensate and quasiparticle contributions [9, 10]. However, key questions remain about both the nature of the phase transition and the mechanism which drives it. Is the transition between a phasecoherent state and an exotic compressible state? [1] Is the transition driven by the percolation of regions which are incompressible? 12] Is the transition weakly firstorder in the absence of disorder? 13. Is the transition associated with softening of the collective-mode at a finite wavevector? 2, 3, 14] How does disorder affect these scenarios? To address these questions, it is necessary to understand the evolution of the two important energy scales, the collective-mode gap at zero wavevector and the roton-minimum gap near $k l \sim 1$, near the phaseboundary. In this Letter we show that noise-spectroscopy of interlayer current probes the collective mode energy at zero wavevector, whereas noise-spectroscopy of charge imbalance probes the roton-like minimum in the collective mode energy. Combined, these two measurements can establish the nature of the transition and shed light on effect of disorder on the transition.

Noise analysis has been used extensively to probe intrinsic properties of many different systems [15]. For example, it can be used to measure the atomic magnetic resonance in a sample without an external noise source 16 and has been suggested as a technique for single-spin detection using a scanning tunneling microscope [17]. In this paper we propose that the same technique can be used to answer key questions in bilayer quantum Hall systems. Although the average current between layers is zero when the system is not driven by an interlayer electrochemical potential difference, spectral analysis of fluctuations in the current can reveal the condensate and quasiparticle energy scales of the phasecoherent state. Current fluctuations of the system are controlled by the current-current susceptibility. In the absence of an electron-hole condensate, this susceptibility is controlled by the incoherent contribution of quasiparticles in the system, and the correlation time of fluctuations will be dominated by the quasiparticle energy gap. In contrast, the appearance of the condensate implies a collective mode which transfers charge from one layer to the other. This collective mode will contribute to the fluctuations; hence in the ordered state we will have a qualitatively different spectrum of fluctuations. A similar analysis applies to the fluctuations in the charge imbalance which lead to fluctuations in the electrostatic potential. These effects are the basis of the noise spectroscopy we propose. We find that the current-current correlator oscillates with characteristic frequency equal to the collective-mode energy at zero wavevector, $\omega_{c}=E_{s w}(\mathbf{0})$ (we use units such that $\hbar=1$ ), if the interlayer transport is predominantly collective. We also find that the electrostatic-potential correlator at long times has a characteristic frequency equal to the collective-mode energy minimum, $\omega^{*}=E_{s w}^{*}$, which occurs at a finite wavevector $k l \sim 1$. Thus noise-spectroscopy of a bilayer system can probe the two important energy scales, $E_{s w}(\mathbf{0})$ and $E_{s w}^{*}$, in the phase-coherent state and their evolution as 
$d \rightarrow d_{c r}$

In the following paragraphs, we describe the microscopic model we use for a biased bilayer system, obtain analytical expressions for the current-current and electrostatic-potential correlators in terms of bilayer response functions, and discuss the implications of these results.

Microscopic Model: Let us consider a biased bilayer system with filling factor $\nu_{T}$ in the top layer and $\nu_{B}$ in the bottom layer such that the total filling factor $\nu=$ $\nu_{T}+\nu_{B}=1$. We use a pseudospin language to describe the bilayer system.

$$
\hat{S}_{\alpha}(\mathbf{r})=\frac{1}{2} \sum_{\sigma^{\prime}, \sigma} c_{\sigma^{\prime}}^{\dagger}(\mathbf{r}) \tau_{\sigma^{\prime} \sigma}^{(\alpha)} c_{\sigma}(\mathbf{r})
$$

is the pseudospin density in direction $\alpha$ at position $\mathbf{r}$, $\sigma, \sigma^{\prime}$ are the pseudospin labels, and $\tau_{\sigma^{\prime} \sigma}^{(\alpha)}$ are Pauli spin matrices with pseudospin up/down representing electrons in top/bottom layers. The microscopic Hamiltonian for the bilayer system is given by $\hat{H}=\hat{H}_{0}+\hat{V}$. The one-body term is

$$
\hat{H}_{0}=-\int_{\mathbf{r}}\left[\Delta_{t} \hat{S}_{x}(\mathbf{r})+\Delta_{v} \hat{S}_{z}(\mathbf{r})\right]
$$

where $\Delta_{t}$ is the interlayer tunneling amplitude and $\Delta_{v}$ is the bias voltage which leads to unequal filling factors in the two layers. The interaction term $\hat{V}$ is a sum of the intra-layer Coulomb interaction, $V_{A}(\mathbf{k})=2 \pi e^{2} / \epsilon k$ and the interlayer Coulomb interaction $V_{E}(\mathbf{k})=e^{-k d} V_{A}(\mathbf{k})$. Note that the antisymmetric combination $V_{x}=\left(V_{A}-\right.$ $\left.V_{E}\right) / 2$ is not rotationally invariant in the pseudospin space. In the pseudospin-language, the phase-coherent incompressible state is an $X Y$ easy-plane ferromagnet and has a uniform non-zero value of $\left\langle\hat{S}_{z}(\mathbf{r})\right\rangle$ when the filling factors in the two layers are unequal [18, 19]. When $\Delta_{t} \neq 0$ the $\mathrm{U}(1)$ symmetry in the $x-y$ plane in pseudospin-space is broken explicitly, and the uniform ordered moment $\vec{M}$ lies in the $x$-z plane, $\vec{M}=$ $M_{0}\left(\sin \theta_{v}, 0, \cos \theta_{v}\right)$ 18]. $M_{0}$ is the dimensionless order parameter which vanishes near the incompressible-state phase-boundary and approaches 1 as the layer separation $d \rightarrow 0$. The angle $\theta_{v}$ is determined by self-consistent Hartree and exchange contributions to the tunneling amplitude and bias voltage, and is given by $\cos \theta_{v}=\Delta_{v} / \Delta_{v c}$ in the limit $\Delta_{t} \rightarrow 0$. Here $\Delta_{v c}(d)=2\left[V_{x}(0)-\Gamma_{x}(0)\right]$ is inverse of the exchange-enhanced interlayer capacitance, $\Gamma_{x}=\left(\Gamma_{A}-\Gamma_{E}\right) / 2$, and $\Gamma_{(A / E)}(\mathbf{k})$ are the intralayer/interlayer Coulomb exchange interactions.

Current Noise: The only term in the microscopic Hamiltonian which does not conserve particle number within each layer separately is proportional to the interlayer tunneling amplitude $\Delta_{t}$. Therefore the total interlayer current $\hat{I}$, obtained by using the continuity equation [20], is given by

$$
\hat{I}=e \Delta_{t} \int_{\mathbf{r}} \hat{S}_{y}(\mathbf{r})
$$

In the absence of any electrochemical potential difference between the two layers the average interlayer current is zero, $\langle\hat{I}\rangle=0$. However, as we will see, the current-current correlations are nonzero. Let us consider the symmetrized current-current correlator, $C_{I}(t)=$ $\langle\hat{I}(t) \hat{I}(0)+\hat{I}(0) \hat{I}(t)\rangle$. Using Eq. (3), it is straightforward to express the correlator in terms of pseudospin response function

$$
C_{I}(t)=\left(e \Delta_{t}\right)^{2} 2 \operatorname{Re} \chi_{y y}(\mathbf{k}=0, t)
$$

where $\chi_{y y}(\mathbf{k}, t) \equiv\left\langle T \hat{S}_{y}(\mathbf{k}, t) \hat{S}_{y}(-\mathbf{k}, 0)\right\rangle$ is the timeordered pseudospin susceptibility. For quantum Hall bilayers, it is possible to calculate the susceptibility analytically from the microscopic Hamiltonian using different approximations which capture either the quasiparticle or the collective-mode physics. Since the interlayer transport in the phase-coherent state is presumably collective [7, 8, 9, 10, we evaluate the susceptibility using generalized random phase approximation (GRPA) which captures the physics of collective modes [2, 4],

$$
\chi_{y y}\left(\mathbf{k}, i \Omega_{n}\right)=\frac{e^{-k^{2} l^{2} / 2}}{8 \pi l^{2}} \frac{2 i M_{0} a_{\theta_{v}}(\mathbf{k})}{\left(i \Omega_{n}\right)^{2}-E_{s w}^{2}(\mathbf{k})},
$$

where $i \Omega_{n}$ is a bosonic Matsubara frequency and the exponential prefactor arises from projection onto the lowest Landau level. The collective-mode dispersion is given by $E_{s w}(\mathbf{k})=\sqrt{a_{\theta_{v}}(\mathbf{k}) \cdot b_{\theta_{v}}(\mathbf{k})}$ where 18]

$a_{\theta_{v}}(\mathbf{k})=\Delta_{q p}-M_{0} \Gamma_{E}(\mathbf{k})+2 M_{0}\left[V_{x}(\mathbf{k})-\Gamma_{x}(\mathbf{k})\right] \sin ^{2} \theta_{v}$

is the cost of charge-imbalance fluctuation and $b_{\theta_{v}}(\mathbf{k})=$ $\Delta_{q p}-M_{0} \Gamma_{E}(\mathbf{k})$ is the cost of phase-fluctuations in the $x-y$ plane. Here, the quasiparticle energy splitting is given by $\Delta_{q p}=M_{0} \Gamma_{E}(0)$ when $\Delta_{t} \rightarrow 0$ or $\Delta_{v} \rightarrow 0$; for a nonzero tunneling and nonzero bias voltage the quasiparticle energy splitting is obtained by solving the self-consistent Hartree-Fock mean-field equations numerically [18].

We emphasize that the pseudospin susceptibility $\chi_{y y}$ is calculated in the presence of finite interlayer tunneling. Therefore the cost of uniform phasefluctuations is non-zero, $b_{\theta_{v}}(\mathbf{0}) \neq 0$, and the collective mode energy at the origin is nonzero, $E_{s w}(\mathbf{0}) \sim$ $\sqrt{\Delta_{t}\left(\Delta_{t}+M_{0} \Delta_{v c} \sin ^{2} \theta_{v}\right)} \neq 0$. When $\Delta_{t} \rightarrow 0$, the static uniform susceptibility (5) diverges as expected in a state which possesses a spontaneous broken symmetry. It is necessary to treat the interlayer tunneling nonperturbatively because the interlayer current operator $\hat{I}$ vanishes at $\Delta_{t}=0$ 10, 21. It follows from Eq. (5) that the current-current correlator is given by

$$
C(t)=\left(e \Delta_{t}\right)^{2} \frac{2 M_{0} a_{\theta_{v}}(\mathbf{0})}{8 \pi l^{2} E_{s w}(\mathbf{0})} \cos \left(E_{s w}(\mathbf{0})|t|\right)
$$

Eq. (7) is the first principle result of the paper. It shows that the current-current correlator oscillates with characteristic frequency $\omega_{c}=E_{s w}(\mathbf{0})$ and its strength is proportional to $M_{0}$. Therefore the noise spectroscopy of 
interlayer current will be able to probe the collective mode energy at zero wavevector. Note that near the incompressible-to-compressible phase boundary, the only parameter in $E_{s w}(\mathbf{0})$ which varies rapidly with $d / l$ is the order-parameter $M_{0}$; the tunneling amplitude $\Delta_{t}$ and the exchange-enhanced inverse capacitance $\Delta_{v c}$ are relatively constant. Therefore, the dependence of the condensate frequency $\omega_{c}$ on $d / l$ will provide a direct measurement of the phase-coherent order parameter $M_{0}$. For typical values of system parameters, $\Delta_{t} \sim 50 \mu \mathrm{K}$ and $\Delta_{v c} \sim 50 \mathrm{~K}$, and balanced bilayers $\left(\theta_{v}=\pi / 2\right)$, we get $\omega_{c} \sim \sqrt{M_{0}}$ $\mathrm{GHz}$ (for example, with $M_{0} \sim 10^{-1}-10^{-2}$, we have $\left.\omega_{c} \sim 100-300 \mathrm{MHz}\right)$.

Electrostatic Potential Noise: Now let us consider the effect of random charge-imbalance fluctuations on the electrostatic potential. We assume that the potential is measured (using, for example, a single-electron transistor) in a plane located at distance $R$ above the top layer [22]. The change in the electrostatic potential $\delta \phi$ produced by a change in the interlayer charge-imbalance is 23.

$$
\delta \phi(\mathbf{k})=2 e V_{x}(-\mathbf{k}) e^{-k R} \delta S_{z}(\mathbf{k})
$$

where $\delta S_{z}(\mathbf{r})$ is the fluctuation in the charge-imbalance at position r. In steady state, the potential fluctuations average to zero $\langle\delta \phi\rangle=0$. However, the potential correlator is non-zero. Let us define $C_{\phi}(r, t)=$ $\langle\delta \phi(\mathbf{r}, t) \delta \phi(\mathbf{0}, 0)+\delta \phi(\mathbf{0}, 0) \delta \phi(\mathbf{r}, t)\rangle$. It follows from Eq. (8) that this correlator is related to the time-ordered pseudospin susceptibility $\chi_{z z}$,

$$
C_{\phi}(r, t)=4 e^{2} \int \frac{k d k}{2 \pi} J_{0}(k r) e^{-2 k R} V_{x}^{2}(\mathbf{k}) 2 \operatorname{Re} \chi_{z z}(\mathbf{k}, t) .
$$

Eq. (9) is the second principle result of this paper. Following the analysis in the current-noise case, the GRPA susceptibility which captures the physics of collective excitations is given by

$$
\chi_{z z}(\mathbf{k}, t)=\frac{e^{-k^{2} l^{2} / 2}}{8 \pi l^{2}} \frac{M_{0} b_{\theta_{v}}(\mathbf{k})}{E_{s w}(\mathbf{k})} \exp \left(-i E_{s w}(\mathbf{k})|t|\right) .
$$

We note that the static uniform $\chi_{z z}$ susceptibility vanishes in the limit $\Delta_{t} \rightarrow 0$, in contrast to the static uniform $\chi_{y y}$ susceptibility (15). It is also worth emphasizing that the potential correlator $C_{\phi}(r, t)$ can be evaluated perturbatively in the tunneling amplitude $\Delta_{t}$, whereas the current-current correlator $C_{I}(t)$ requires a non-perturbative calculation. First let us concentrate on the short-time behavior of the correlator function $C_{\phi}(r, t)$. It follows from Eq. (10) that the correlator saturates at $t=0$ and falls off quadratically with $t$ at short times $|t| \ll \Delta_{q p}^{-1}$. It is primarily sensitive to the phasefluctuations cost $b_{\theta_{v}}(\mathbf{k})$, which does not change qualitatively as a function of increasing $d / l$. Figure 1 shows the typical $r$-dependence of the potential correlator $C_{\phi}(r, t)$ at short-times for different values of $d / l$.

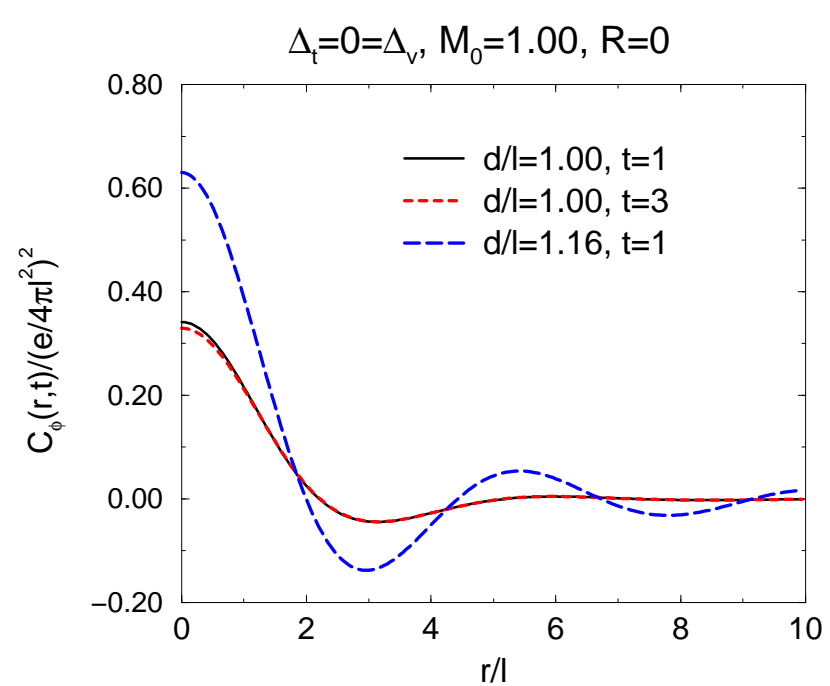

FIG. 1: Typical potential correlator $C_{\phi}(r, t)$. Time is measured in units of inverse Coulomb energy. $C_{\phi}(r, t)$ decays with $r$ due to the Bessel function $J_{0}(k r)$ in the integrand (9), and is not very sensitive to the softening of the roton-like minimum near $k l \sim 1$ which occurs at $d_{c r} / l=1.18$ [2, 3, 4].

To simplify the discussion of the long-time correlator, we will specialize to a bilayer system with $\Delta_{t} \rightarrow 0$ and consider only local correlations, $r=0$, although the results obtained here are valid in general. We remind the Reader that the approximation $\Delta_{t}=0$ is justified when evaluating the potential correlator $C_{\phi}(r, t)$. Restricting to a local correlator corresponds to the usual experimental situation with one single-electron transistor probe 22] which measures (the noise in) the electrostatic potential. At long times, the integral in Eq. (9) is dominated by the region in momentum-space where the pseudospin-wave energy is minimum. It is known that in bilayer systems the collective-mode dispersion typically has two minima [2, 3, 4]. The first minimum occurs at the origin, $\mathbf{k}=0$, and the collective mode dispersion in the vicinity is linear. The second minimum occurs near $k l \sim 1$. The collective-mode energy at this minimum, $E_{s w}^{*}(d)$, reduces as the ratio $d / l$ is increased, due to the lowered energy cost for charge-imbalance fluctuations, $a_{\theta_{v}}(k l \sim 1) \rightarrow 0$. It follows from Eq. 110 that the region near the second minimum gives a diverging contribution, $\chi_{z z} \sim 1 / \sqrt{a_{\theta_{v}}} \rightarrow \infty$, and that contribution varies strongly with layer separation $d / l$. Thus, the longtime potential correlator $C_{\phi}$ has a component with frequency $\omega^{*}=E_{s w}^{*}(d)$ which varies rapidly with $d / l$. The strength of this feature in the potential correlator scales as $\sqrt{M_{0} /\left(d-d_{c r}\right)}$. The incompressible-to-compressible phase transition which occurs at a critical layer separation $d_{c r}$ can be understood in terms of the collapse of this collective-mode energy minimum, $E_{s w}^{*}\left(d_{c r}\right)=0$ 2, 3]. A measurement of the characteristic frequency $\omega^{*}$ and its dependence on $d / l$ can directly probe the collective-mode energy gap collapse and shed light on whether it is in- 
strumental to the phase transition [14]. The variation of $E_{s w}^{*}(d)$ with $d / l$ can also provide an independent $d i$ rect measure of the order parameter $M_{0}$, since close to the phase-boundary the minimum collective-mode energy can be approximated as $E_{s w}^{*}(d) \propto \sqrt{M_{0}\left(d-d_{c r}\right)}$.

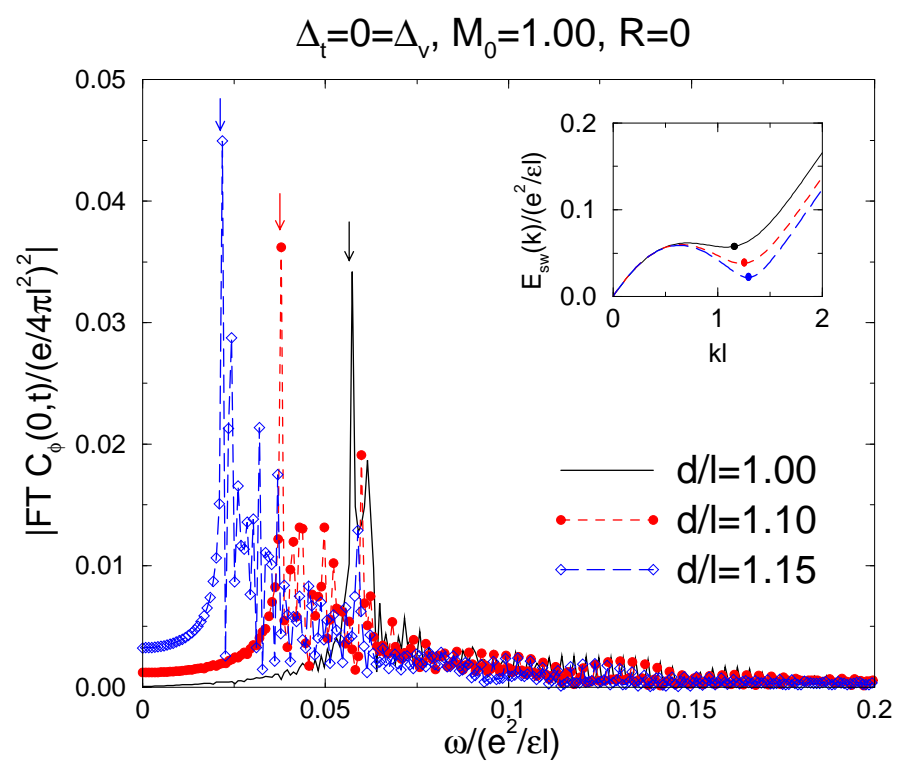

FIG. 2: Fourier transform (FT) of the potential correlator $C_{\phi}(0, t)$. The inset shows the collective-mode dispersions for corresponding values of $d / l$. The peak in the FT, marked by arrows, occurs at $\omega=E_{s w}^{*}(d)$ and varies with $d$ as $\sqrt{\left(d-d_{c r}\right)}$; it probes the collective-mode minimum near $k l \sim 1$, marked by solid circles, shown in the inset.

Figure 2 shows the Fourier transform of the long-time correlator $C_{\phi}(0, t)$ for different values $d / l$, whereas the inset shows the corresponding collective-mode dispersions. The peak near $\omega /\left(e^{2} / \epsilon l\right) \sim .06$ is not sensitive to $d / l$ and represents the contribution from region near the origin where the collective-mode dispersions for all values of $d / l$ are essentially the same. The second peak occurs at $\omega=\omega^{*}=E_{s w}^{*}(d)$ and varies rapidly with $d / l$. The strength of this frequency component increases when $d \rightarrow d_{c r}$ as expected from Eq. (10) 24].

Summary: We have shown that spectral analysis of fluctuations in bilayer quantum Hall systems provides a novel probe of the phase-coherent state. We predict that the current-noise spectrum has a characteristic frequency $\omega_{c}=E_{s w}(\mathbf{0})$ if the interlayer transport is collective. We also predict that the noise in the electrostatic potential has a dominant frequency component equal to the collective-mode energy minimum at finite wavevector, $\omega^{*}=E_{s w}^{*}$. These two measurements can probe directly the order-parameter of the phase-coherent state as well as the collapse of the collective-mode energy gap $E_{s w}^{*}$ near the incompressible-to-compressible phase-boundary. Our theoretical analysis of these quantities does not account for the influence of disorder and inhomogeneity, and does not allow for the possibility that exotic correlated states appear on either side of the phase transition. Although the analysis of the noise spectra in these scenarios is more complex than in the BCS-like excitonic condensate case considered here, it is clear that there will be substantial differences, especially as the phase boundary is approached. Experimental tests of the predictions made above, which we regard as a starting point for the interpretation of experiment, will shed light on the nature of the compressible-to-incompressible phase-transition, and deepen our understanding of the phase-coherent state in quantum Hall bilayers.

It is a pleasure to thank Z. Nussinov for useful discussions. This work was supported by DOE LDRD (LANL), by the NSF under grant DMR0115947 (UT), and by the Welch Foundation (UT).

[1] S.Q. Murphy et al., Phys. Rev. Lett. 72, 728 (1994).

[2] H.A. Fertig, Phys. Rev. B 40, 1087 (1989).

[3] A.H. MacDonald et al., Phys. Rev. Lett. 65, 775 (1990).

[4] K. Moon et al., Phys. Rev. B 51, 5138 (1995); K. Yang et al., Phys. Rev. B 54, 11644 (1996).

[5] S.M. Girvin and A.H. MacDonald in Perspectives in Quantum Hall Effects, edited by S. Das Sarma and Aron Pinczuk; S. Das Sarma and E. Demler, Solid State Commun. 117, 141 (2001).

[6] Yu.E. Lozovik and V.I. Yudson, Solid State Commun. 19, 39 (1976); Y. Kuramoto and C. Horie, Solid State Commun. 25, 713 (1978); D. Paquet et al., Phys. Rev. B 32, 5208 (1985); A.H. MacDonald and E.H. Rezayi, Phys. Rev. B 42, 3224 (1990); D. Yoshioka and A.H. MacDonald, J. Phys. Soc. Jpn. 59, 4211 (1990).

[7] I.B. Spielman et al., Phys. Rev. Lett. 84, 5808 (2000); Phys. Rev. Lett. 87, 036803 (2001).

[8] M. Kellogg et al., Phys. Rev. Lett. 88, 126804 (2002); Phys. Rev. Lett. 90, 246801 (2003).

[9] L. Balents and L. Radzihovsky, Phys. Rev. Lett. 86, 1825 (2001); A. Stern et al., ibid., 1829 (2001); M.M. Fogler and F. Wilczek, ibid., 1833 (2001).

[10] Y.N. Joglekar and A.H. MacDonald, Phys. Rev. Lett. 87, 196802 (2001).

[11] E. Demler et al., Phys. Rev. Lett. 86, 1853 (2001).

[12] A. Stern and B. I. Halperin, Phys. Rev. Lett. 88, 106801 (2002).

[13] J. Schliemann et al., Phys. Rev. Lett. 86, 1849 (2001).

[14] S. Luin et al., Phys. Rev. Lett. 90, 236802 (2003).

[15] E.B. Aleksandrov and V.S. Zapasskii, Zh. Eksp. Teor. Fiz. 81, 132 (1981); D.D. Awschalom et al., Phys. Rev. Lett. 68, 3092 (1992); Y. Manassen et al., Phys. Rev. B 6116223 (2000).

[16] T. Mitsui, Phys. Rev. Lett. 84, 5292 (2000).

[17] Y. Manassen et al., Phys. Rev. Lett. 62, 2531 (1989); Y. Manassen, J. Magn. Reson. 126, 133 (1997); C. Durkan and M.E. Welland, App. Phys. Lett. 80, 459 (2002); J.X. Zhu and A.V. Balatsky, Phys. Rev. Lett. 89, 286802 (2002); A.V. Balatsky et al., Phys. Rev. B 66, 195416 (2003); Z. Nussinov et al., Phys. Rev. B 68, 085402 (2003). 
[18] Y.N. Joglekar and A.H. MacDonald, Phys. Rev. B 65, 235319 (2002).

[19] E. Tutuc et al., Phys. Rev. Lett. 91, 076802 (2003).

[20] In typical samples, current measured in the leads is the sum of local current densities. Therefore, we consider the correlator for the total current instead of current density.

[21] H.A. Fertig and J.P. Straley, Phys. Rev. Lett. 91, 046806 (2003).

[22] A. Yacobi et al., Solid State Commun. 111, 1 (1999);
N.B. Zhitenev et al., Nature (London) 404, 473 (2000).

[23] Strictly speaking, this expression is valid for an infinite sample with dielectric constant $\epsilon$; however, results obtained here are unchanged when we take into account the dielectric constant discontinuity at the sample surface.

[24] These results remain valid even if the phase transition is driven by reducing the bias voltage [18, 19] instead of increasing $d / l$. 